\title{
Ransomware Targeting Automobiles
}

\author{
Pranshu Bajpai \\ Michigan State University \\ East Lansing, Michigan, USA \\ bajpaipr@msu.edu
}

\author{
Richard Enbody \\ Michigan State University \\ East Lansing, Michigan, USA \\ enbody@cse.msu.edu
}

\author{
Betty H.C. Cheng \\ Michigan State University \\ East Lansing, Michigan, USA \\ chengb@msu.edu
}

\begin{abstract}
Ransomware has consistently been the top threat to security in recent years. It seems inevitable that ransomware developers will ultimately target automobiles. As vehicles get smarter and more connected, the threat surface widens and automobiles naturally become more vulnerable to Internet-based threats such as ransomware. In this paper, we identify the constraints on ransomware in the context of automobiles and demonstrate potential ransomware attacks. The demonstrations entail attack vectors targeted at the IVI system and the methodology for implementing a hybrid crypto ransomware on an IVI system. Finally, we clarify the new ransomware strategies that the attackers could leverage in the context of vehicular systems with the intent to promote research to prevent such attacks.
\end{abstract}

\section{CCS CONCEPTS}

- Security and privacy $\rightarrow$ Malware and its mitigation; Denialof-service attacks.

\section{KEYWORDS}

automotive security, ransomware, denial-of-service

\section{ACM Reference Format:}

Pranshu Bajpai, Richard Enbody, and Betty H.C. Cheng. 2020. Ransomware Targeting Automobiles. In Proceedings of the Second ACM Workshop on Automotive and Aerial Vehicle Security (AutoSec '20), March 18, 2020, New Orleans, LA, USA. ACM, New York, NY, USA, 7 pages. https://doi.org/10. $1145 / 3375706.3380558$

\section{INTRODUCTION}

Internet-of-Things (IoT) systems continue to grow and the automotive industry plays a significant role in this trend [14]. As vehicles become "smarter" with increasingly sophisticated features that involve both onboard and outward facing communication, a new threat surface has emerged for automobiles that must be thoroughly assessed for potential vulnerabilities. In the wake of the highly synergistic Ransomware-as-a-Service (RaaS) underground, smart automobiles need preemptive protection against potential ransomware attacks. Unlike other application domains plagued by cybersecurity crime (e.g., financial systems and privacy data), financial motivations for cybercrime are not obvious, except in the

Permission to make digital or hard copies of all or part of this work for personal or classroom use is granted without fee provided that copies are not made or distributed for profit or commercial advantage and that copies bear this notice and the full citation on the first page. Copyrights for components of this work owned by others than ACM must be honored. Abstracting with credit is permitted. To copy otherwise, or republish, to post on servers or to redistribute to lists, requires prior specific permission and/or a fee. Request permissions from permissions@acm.org.

AutoSec '20, March 18, 2020, New Orleans, LA, USA

(C) 2020 Association for Computing Machinery.

ACM ISBN 978-1-4503-7113-1/20/03 . \$ \$15.00

https://doi.org/10.1145/3375706.3380558 context of ransomware attacks. Potential vulnerabilities in the automobile systems, combined with the tenacity of malware developers, makes automobile security against ransomware a challenging problem. In an attempt to highlight vulnerabilities and motivate research to prevent breaches, this paper describes our efforts to clarify the automobile ransomware kill chain, identify potential attack vectors, and illustrate ransomware vulnerability for targeted data and services on automobile In-Vehicle Infotainment (IVI) systems.

Malware developers are known to be opportunistic in exploiting improperly secured systems to infect these vulnerable devices with malware. For instance, the infamous WannaCry ransomware exploited multiple vulnerabilities in Message Block 1.0. In another example, the Mirai botnet infected over 600, 000 vulnerable IoT devices at its peak [24] [4]. In parallel, remote exploitation of automobiles has been demonstrated [31]. It seems inevitable that ransomware developers target automobiles and yet there is a lack of research on systematic evaluation of the associated risk. In light of these realizations, it is incumbent that we recognize and eliminate the attack vectors in modern automobiles before malware developers seek to exploit them.

In this paper, we highlight the constraints that ransomware developers face while attacking automobile systems, where the information was then used to enumerate data and services of potential interest to ransomware developers. After an extensive review of the state of the art automotive cybersecurity vulnerabilities and techniques, complemented with feedback from automotive industrial partners, we were able to better understand the motivation, likelihood, and impact of ransomware attacks on an automobile IVI system. With this knowledge, we performed several experiments that simulated attacks on an IVI system and studied the outcomes.

Our results show that it is viable for ransomware developers to implement effective ransomware attacks by satisfying all constraints in a kill chain in automobiles. The abstraction that ransomware developers seek is present in the form of dynamic libraries on the IVI systems. Implementing cryptographic functionality then becomes trivial for malware developers. The attack vector most likely to be exploited is configuration and authentication oversights in the IVI systems and ransomware will target not just denial-ofdata in the IVI, but also denial-of-service (denial of resources) and denial-of-privacy to gain sufficient leverage over the victim.

We performed experiments to exercise these vulnerabilities. Specifically, we tested a proof-of-concept crypto-ransomware that encrypts data on a QNX-based IVI system (denial of data). We also tested resource exhaustion by creating a fork bomb on the QNXbased IVI (denial of service). And finally, we tested exploitation of configuration errors by gaining execution via an exposed QCONN service on an IVI system. We were able to confirm the adverse consequences of these attacks by measuring system resource usage before and after the simulated attacks. The remainder of this paper 
is organized as follows. Motivations for investigating ransomware for the automotive domain are highlighted in Section 2. The differences between cybersecurity challenges facing the automotive domain and the IT sector are overviewed in Section 3. With these differences in mind, we next describe how ransomware developers might exploit the automobile vulnerabilities in Section 4. We describe our approach and experimental setup for exploring these vulnerabilities within the context of an IVI system in Section 5 and present experimental results in Section 6. Finally, we provide conclusions and discuss future work in Section 7.

\section{RELATED WORK}

The threat of malware in the automotive domain has been previously considered [43]. The need for rethinking modern automobile platforms to incorporate security with the existing notion of safety has been acknowledged [10]. While these papers acknowledge the looming threat of malware over automobiles, there has been no prior work on explicitly studying the constraints on ransomware developers in the context of automobiles. To the best of our knowledge, we are not aware of any prior studies done to simulate a ransomware attack on a vehicular system to identify just how viable certain attack vectors are and if ransomware on automobiles is an immediate or near future concern in the realm of automobile security. However, Richardson et al. [35] acknowledge that as the world moves to IoT, a ransomware strike on IoT is imminent. The security industry similarly expects the rise of "ransomware-of-things" which will inevitably affect automobiles [8]. Yaqoob et al. [42] highlight the lack of security controls in resource-constrained IoT devices and the challenges posed by ransomware in the IoT space. The same challenges also plague the automotive environment with several automotive components viewed as IoT devices. Finally, Wolf et al. [41] discuss attack paths that could lead to successful ransomware attacks on automobiles.

\section{DIFFERENCES BETWEEN AUTOMOBILE AND TRADITIONAL IT SECURITY}

Automobile security is made particularly challenging by the fact that many of the traditional IT security concepts cannot be directly applied to vehicles due to several fundamental differences between vehicles and traditional computers. A summary of these differences is presented in Table 1. In summary, automobiles have longer life spans, relatively new security standards, new and potentially insecure IoT components, limited resources for implementing security controls, and real-time performance constraints.

\section{RETHINKING RANSOMWARE}

Traditional ransomware attacks target data residing on computer systems and perform unauthorized encryption of user files. This encryption is performed using a unique secret, a key known only to the attacker, and standard encryption algorithms such as AES and RSA [5]. A symmetric encryption algorithm is deployed for fast bulk data encryption, while an asymmetric encryption algorithm is used to protect the secrecy of the symmetric key until the ransom is paid. Attackers often deploy dynamic cryptographic libraries available on host to perform the encryption [33] and subsequently demand a ransom payment. Targeted ransomware attacks are gaining prominence indicative of the strategic decision-making deployed by the attackers towards profit maximization.

In light of the differences between the traditional computing environments and the automotive domain, ransomware developers will need to rethink certain strategies. The attacker's view of the automobile platform is shown in Figure 1. Internal and external filters as shown in Figure 1 pertain to security controls in place to prevent unauthorized access. Ideally, both of these controls should prevent the attacker from accessing the automobile subsystems. However, the internal filter should be more restrictive and hardened than the external filter to ensure an infection does not crossover from the IVI system to the internal CAN. To this effect, air-gapping [9] the IVI from the CAN is the best strategy. This ensures that a malware infection on the IVI cannot severely debilitate the vehicle by accessing the CAN. However, if such air-gapping is infeasible in the interest of functionality, and communication between IVI and CAN is necessary, this communication should be minimized and thoroughly validated to ensure malicious input from the IVI does not crossover to the CAN. In other words, the vulnerable internal network, CAN, should be treated as ring 0 , while the more exposed IVI system running user applications can be treated as ring 2 according to the Multics rings of protection [28].

Propagation to other areas that communicate with the infotainment system is theoretically possible, however, research is needed to comprehend the real probability of an IVI to CAN (or other subsystems) crossover infection. Even if the malware is able to bypass the internal filter, it is now in a completely different architectural environment and thus needs to be cross-platform to operate. The complexity involved in such a ransomware attack is extreme and a large subset of ransomware developers are known to engage in cargo-cult programming [18]. Furthermore, studies have shown that $94 \%$ of ransomware seen in the wild are ineffective scareware [23]. Hence, the complexity of breaking out of the infotainment and spreading to more critical subsystems and networks needs to be carefully studied for a realistic risk assessment. In this paper, we focus only on the intricacies of a ransomware attack that bypasses the external filter and is able to execute on the IVI system.

\subsection{Attack vectors}

Traditionally, ransomware - and malware threats in general - have depended on phishing as their primary attack vector [39]. A security chain is only as strong as its weakest link and studies have shown that humans often prove to be the weakest link in the security chain [30]. However, sending a phishing email to a user on an IVI system is not feasible since the IVI systems are not typically designed for opening email attachments. The ransomware developers hence need to rethink their attack vectors.

During our experimentation, we discovered exploitation of configuration errors, brute forcing of remote login services, and exploitation of known code execution vulnerabilities in components to be viable attack vectors for ransomware developers in the context of automotive security. These attack vectors are detailed in Section 6 . 
Table 1: Differences in automobile and traditional IT security

\begin{tabular}{lll}
\hline Metric & Automobile Security & Traditional IT Security \\
\hline $\begin{array}{l}\text { Primary concern } \\
\text { Standards }\end{array}$ & Protecting human lives & Protection against losses resulting from breaches \\
Life span & ISO/SAE 21434 is relatively new [38] & ISO27001 is well-established [6] \\
Updates & Up to 15 years [20] & Much shorter life span \\
Network security & Lack of regular Over-The-Air (OTA) updates & Regular OTA updates and patch management cycles \\
& No authentication and no confidentiality in & Provisions for authentication and confidentiality \\
CAN broadcasts [40] & Designed with isolated Controller Area Net- & Designed with interconnectivity in mind \\
wesk (CAN) in mind & Limited memory and processing capabilities & Greater processing power and memory \\
Impact of unavailability & Life threatening & Financial, reputation, or informational losses \\
Blackbox security assessment & Difficult due to proprietary technology & $\begin{array}{l}\text { Easier since most technologies are well documented } \\
\text { and publicly accessible }\end{array}$ \\
& Need to be highly resource efficient & $\begin{array}{l}\text { More computing power means security solutions are } \\
\text { not as constrained }\end{array}$ \\
\hline
\end{tabular}

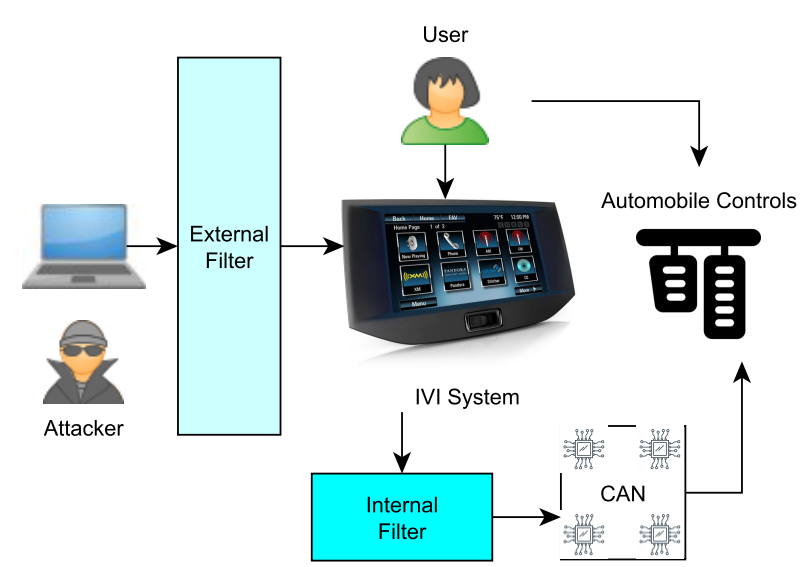

Figure 1: Attacker's view of the automobile platform.

\subsection{Attack focus}

In the vehicular domain, ransomware needs to rethink the strategy of primarily implementing a denial-of-data attack on the host by means of encrypting user files. This is because while in the case of a computer the user has irreplaceable files on the system, the replaceability of user data on an IVI may be high. Hence, in order to gain leverage over victims, automobile ransomware will seek to implement the following types of unavailability attacks on hosts.

Denial-of-data. As discussed above, the data that is resident on an infotainment system may or may not provide enough leverage for the ransomware to successfully trigger a ransom payment from the car owner. Typical ransom demand in the case of traditional ransomware has been approximately $\$ 300$ [17]. In the absence of irreplaceable personal data on the IVI, a ransom demand of $\$ 300$ or more seems unlikely. Perpetrators will need to either locate critical data on IVI or target services (functionality) and privacy instead.
Denial-of-service. This attack corresponds to making the system resources - such as processing power, memory, disk space, and system functionality - unavailable to the user. Therefore, this attack could also be referred to as a denial-of-device. Resource exhaustion could be accomplished by, for example, a fork bomb [32] implemented on the system. An instance of this fork bomb as executed on the vehicular IVI system is shown in Listing 1 . A resource exhaustion attack, such as the fork bomb, renders the IVI system unusable. The system could be rebooted but a ransomware can run such fork bombs after every reboot until the ransom demand is met. In another form of denial of service, a ransomware can push an obstructive ransom screen on the IVI display such that the user is unable to access the IVI functionality.

Denial-of-privacy. The private information discovered on the infotainment system can be used towards extortion [13]. If the system lacks enough private information at the time of infection, the ransomware can lie dormant until private data can be collected. For example, cell phones connected to the IVI systems can transfer private data such as call records, contacts, text messages, etc. to the IVI system [21]. In addition, Global Positioning System (GPS) location data can be held for ransom.

\section{Listing 1: A fork bomb for IVI.}

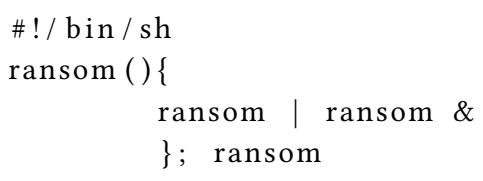

\section{RESEARCH METHODOLOGY}

In this section, we describe our approach to exploring and demonstrating vulnerabilities of interest to ransomware that target the automobile platform. First, we map the likely attack vectors for an automobile ransomware. Then we demonstrate the potential impact of simulated ransomware attacks on an IVI system. These simulated attacks enable us to observe and collect a set of constraints on 
ransomware in the automotive domain, as well as illuminate other useful insights for developing attack prevention strategies.

Experimental setup. We used the test IVI bench as shown in Figure 2. The IVI bench shown in Figure 2 runs on the QNX [19] [26] Real-Time Operating System (RTOS). QNX is a popular soft RTOS powering the IVI systems in a variety of modern cars. For redundancy, experiments were also performed on the VMWare image of QNX available on the QNX official website [1]. Furthermore, a Kali Linux host was used to perform blackbox penetration tests on the QNX-based IVI. Our goal of this setup was to map the threat surface on a QNX-based IVI system and identify the attack vectors that will appeal to our adversaries. Once an attack vector is successfully exploited to gain code execution privileges, we identified the constraints under which ransomware developers will operate their malware. Although this methodology is demonstrated for the QNX RTOS, it can be applied to other powering IVI systems.

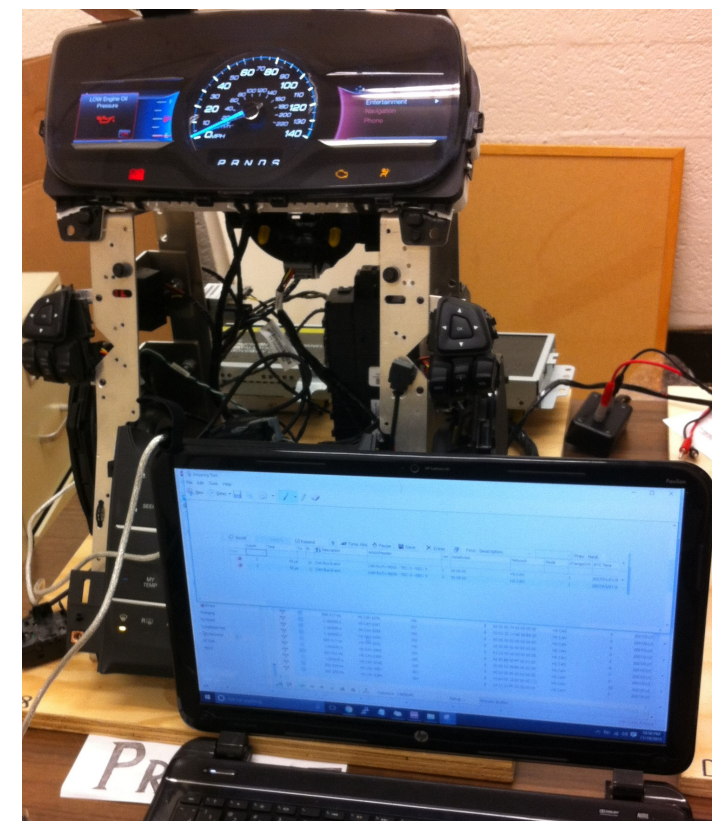

Figure 2: Experimental setup.

The following is a list of the key experiments performed on QNX target box to simulate a ransomware attack:

- Vulnerability analysis of the QNX Neutrino RTOS to discover potential attack vectors.

- Encryption and decryption of data files under the most resilient hybrid cryptosystem observed in modern ransomware that deploys a combination of symmetric and asymmetric encryption [5].

- Simulation of a fork bomb to exhaust system resources.

\subsection{Attack vectors}

Our methodology for discovering viable attack vectors on the QNX target box consisted of a blackbox security assessment [34] of the QNX surface. The following steps were observed during this assessment:
- We used a network mapping utility (nmap) to scan for the IP address of the target box on the subnet. QNX was discovered to be listening on a static IP: 192.168.1.26.

- nmap is used once again to map the available open ports on the target QNX box. A number of services were discovered to be listening for connections on the box (Figure 3).

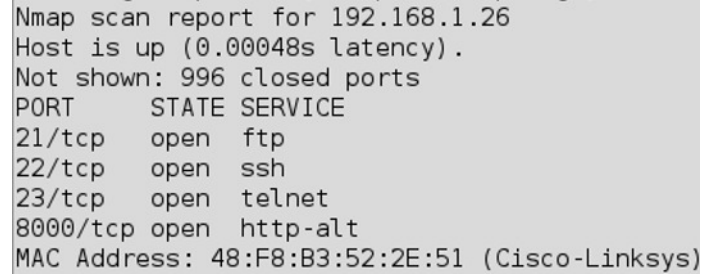

Figure 3: Ports open on the QNX box.

- All login attempts to ports 22 and 23 using a list of default credentials failed. However, depending on the configuration of SSH and Telnet, an attacker might to able to successfully capitalize on this attack vector similar to Remote Desktop Protocol (RDP) being exploited by ransomware in the traditional computing environments.

- Next, port 8000 was investigated and netcat identified a QCONN service listening on port 8000 . QCONN was discovered to be a service that allows connecting the Momentics IDE development platform on a host to the target execution system running QNX Neutrino OS. The QNX official documents identify QNX as "inherently insecure and is meant for development systems only". Furthermore, there are plans to provide QCONN a security model with some form of authentication in the future [1]. However, in its current state, QCONN makes a system vulnerable to arbitrary code execution as shown below. Note that QNX is a true micro-kernel and all services, including security, are add-ons. Hence, it is the responsibility of the automobile manufacturer to configure QNX securely.

- The debugging tool gdb is available for download from the QNX website [1] and allows running unauthenticated applications on the target QNX environment (Figure 4).

(gdb) target qnx 192.168.1.26:8000

Remote debugging using 192.168.1.26:8000

MsgNak received - resending

Remote target is little-endian

(gdb) upload nk-arm-android-1 $7 / \mathrm{tmp} / \mathrm{nk}$

(gdb) run /tmp/nk - lvp 1337 -e /bin/sh

Starting program: /tmp/nk -lvp 1337 -e /bin/sh

Remote: /tmp/nk

[New pid 5460037 tid 1]

Figure 4: GDB allows remote code execution via QCONN.

We have since noticed that this vulnerability has been independently discovered before [2] and that QCONN should be disabled in the production environment. However, configuration errors could cause this service to be exposed in production systems. 
- We are now able to connect to the QCONN service using netcat and executing uname -a identifies QNX Neutrino version 6.5.0 running on the IVI system.

\section{RESULTS}

During the course of experimentation, we noticed how automotive ransomware will differ from traditional ransomware and identified a set of constraints on the vehicular ransomware. We also highlight the most likely attack vectors that will be exploited by malware developers to infect IVI systems.

\subsection{Traditional ransomware versus vehicular ransomware}

It was observed that ransomware on vehicles will differ from standard ransomware in the following ways:

- Automobiles typically have limited computing resources thus making them more vulnerable to denial of service attacks.

- Users have a more immediate need of "service" from vehicular systems than traditional computers since Personal Computer (PC)s can be substituted with another until the ransomware threat is neutralized. In the traditional computing scenario, generally it's the data that is the most crucial to the user and hence held for ransom, not the computing device itself. However, in the case of vehicular infotainment system, the device and its services become a prominent attack target among other items such as the data.

- In the absence of irreplaceable data on a vehicle's infotainment system, ransomware will seek other means of extortion. This includes threatening to release discovered private information or exhausting infotainment system's resources such that it no longer responds.

- Unavailability of an infotainment system can be achieved by simply flashing a screen on the entire display such that it cannot be closed or circumvented by a user.

- Attacks on the infotainment system demand a more immediate response since the driver cannot afford even momentary distractions. This real-time constraint imposes a sense of urgency not as easily achieved in traditional computing environments.

- Perpetrators will need to adjust the ransom business model such that the ransom amount is lowered to make it easier for a car owner to pay the ransom amount (e.g. \$20) than take the vehicle to a dealership to get ransomware removed and get the system reinstated. This does not deter ransomware operators since even a small ransom amount can be multiplied into $N$ number of vehicles which ensures a hefty ransom. For instance, upon infecting the Ford F-series, the multiplier, $N$, will be of the order of 300,000 even if the attack only impacts a single year's models.

- Ransomware can reasonably assume no protections such as antiviruses or firewalls on an infotainment system as opposed to traditional computing equipment which is much better protected. This implies IVI systems are more vulnerable if an attack vector is successfully exploited and the infection infiltrates the defenses. Due to the absence of an antivirus, ransomware execution will not be hindered.

- Unlike traditional computers, infotainment systems on vehicles will be running on different operating systems such as QNX, Windows Embedded Automotive 7, GENIVI, Android, etc [3] [29] [15]. This means a ransomware developer will only be able to write a ransomware that targets a specific subset at a time. In contrast, traditional ransomware have targeted mostly PC systems. Attack vectors will vary depending on the vulnerabilities discovered on these varied IVI operating systems.

We also observed the following set of constraints on vehicular ransomware during the experimentation.

6.1.1 Constraints on automobile ransomware. Constraints presented below indicate certain conditions that must be true for the ransomware to attain its ultimate objective of producing financial rewards for the perpetrators. These constitute the ransomware kill chain or the path our adversary must take to succeed. The following set of constraints hold true for automobile ransomware:

- C1. Penetrating the host's defenses

- C2. Gaining execution privileges on the host

- C3. Enumerating data and services to be ransomed

- C4. Establishing a cryptographic secret (in case of data encryption)

- C5. Protecting the cryptographic secret until ransom is paid

- C6. Removing user's access to needed data and/or services

- C7. Establishing a functional ransom payment route

Some of these constraints are self-explanatory and are axioms of the malware realm. For instance, hosts that are secure enough to deny entry to a malware threat remain impervious to that malware threat. Ransomware are known to deploy a variety of attack vectors to infiltrate the host. These attack vectors include phishing [11], exploiting known vulnerabilities [27], and brute forcing weak RDP sessions [12].

A unique cryptographic secret (a key) needs to be established by crypto-ransomware so that victims of the campaign cannot share keys once a victim pays the ransom and obtains the key.

\subsection{Attack vectors}

An attack vector is defined as the path an attacker takes to circumvent system controls and compromise a host [37]. The following attack vectors emerged after a blackbox assessment [34] was done during the experimentation.

Exploitation of configuration errors. Existing vulnerabilities or configuration errors in the operating system can lead to the compromise of the IVI system.

Brute forcing remote login services. Ransomware are known to exploit poorly secured RDP sessions in order to infiltrate hosts. This can be extended to other remote login services such as SSH and Telnet as discussed previously.

Known vulnerabilities on exposed components. After-market OBD devices promise additional functionality, but can also severely increase the threat surface. For instance, a dongle that connects to the Internet when plugged into the OBD2 port can be exploited 
remotely by exposing vulnerabilities that exist in the firmware or configuration. Moreover, vulnerabilities in the RTOS that power the IVI can also permit remote attacks.

\subsection{Denial of data}

Similar to traditional ransomware, vehicular ransomware can achieve a denial of data attack by encrypting the data with a secret key and then purging the original copy. The most potent Category 6 ransomware [5] have been observed to implement this attack by deploying a hybrid cryptosystem that utilizes both symmetric and asymmetric encryption for their respective advantages as discussed in Section 4.

The availability of openssl, a suite of cryptographic tools [36], on the QNX RTOS facilitated the implementation of the hybrid cryptosystem. On other IVI systems, attackers may deploy another cryptographic library to achieve data encryption. The following series of steps were performed to implement a hybrid cryptosystem in the ransomware:

- Attacker begins by generating a set of asymmetric RSA-2048 keys. The public key is embedded in the ransomware binary and ships with the ransomware infection.

- Next, the ransomware binary on the infected host is able to generate a new AES key that will be used as an encryption key for the purpose of encrypting files.

- The generated AES symmetric key can now be deployed for bulk data encryption. The data file is successfully encrypted.

- The attacker's embedded RSA public key is used to encrypt the encryption key. Note that the RSA private key never left the attacker.

- The original data file is now purged from the system, along with the unencrypted AES key. A ransom note is displayed to the user.

- After successful ransom payment, the process is reversed. The attacker decrypts the encrypted AES key using their RSA private key.

- The decrypted AES key is returned to the user which can now be used to decrypt data.

The summarized sequence of encryption and decryption commands is shown in Listing 2.

\section{Listing 2: OpenSSL-based ransomware in automobiles.}

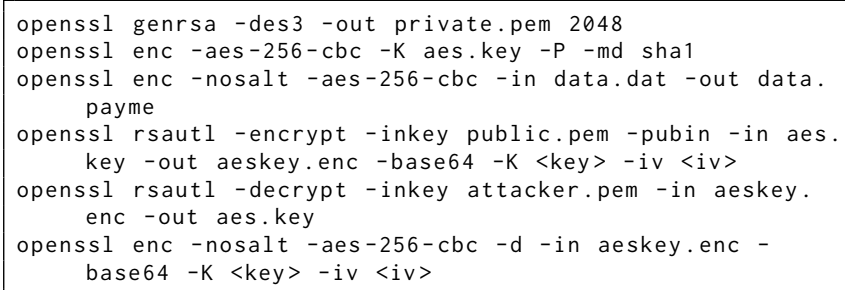

\subsection{Denial of service}

A denial of resources was achieved in the experimental setup in the following manner. The QNX target was infected with a fork bomb shown in Listing 1 . The results of this infection were a complete exhaustion of QNX system resources, namely CPU and memory. Comparison of the system state before and after the execution of the fork bomb is shown in Figure 5. The number of processes and threads peaked at maximum load capacity immediately after execution of the fork bomb such that CPU idle percentage was observed drop from $99 \%$ to $0 \%$ and the memory available dropped from $182 \mathrm{MB}$ to $40 \mathrm{MB}$. This is because the form bomb caused a dramatic increase in redundant processes such that the process count rose from 40 to 194 until complete resource exhaustion.

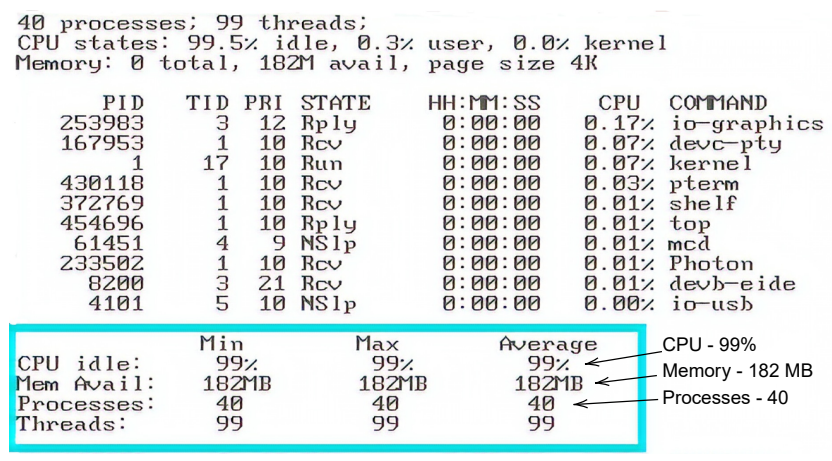

(a) Before

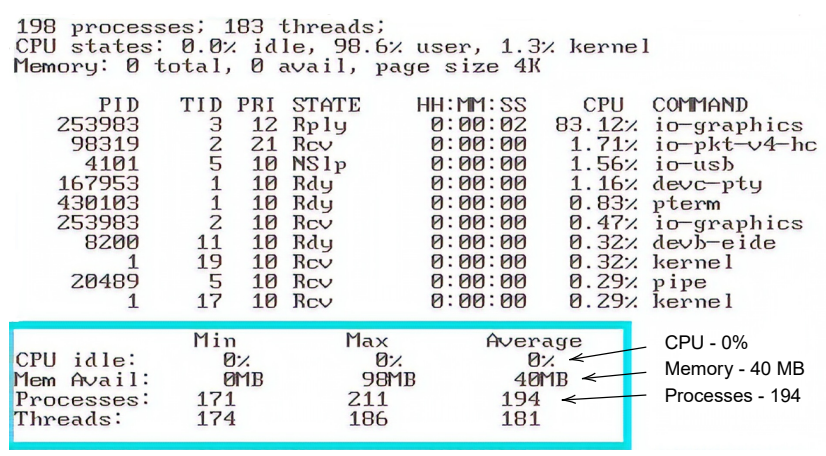

(b) After

Figure 5: Denial of service realized with a fork bomb.

\section{CONCLUSIONS AND FUTURE WORK}

This paper explores ransomware attacks in the context of the automotive domain. We identified the ways in which a ransomware threat on a vehicular surface will differ from that seen in the traditional computing environment. To the best of our knowledge, the threat of ransomware on a vehicular IVI has not been previously systematically analyzed. By experimenting with the simulation of a ransomware attack on an IVI, we were able to clarify the automobile ransomware kill chain and the corresponding impact on the automobile systems. Although we have demonstrated resource exhaustion and data encryption attacks for the QNX platform, these are applicable to other IVI operating systems due to the lack of inherent protections against malicious activity in most RTOS. 
Our future work will explore a number of follow-on investigations including tests on other operating systems such as INTEGRITY and ThreadX. Moreover, we will extend these experiments to include real-world settings using our ongoing collaborations with both automotive OEM and suppliers. While IVI make use of external networks, we will explore other vulnerable automotive attack surfaces that have been identified as vulnerable to remote and local attacks from malicious sources [16] [25]. We have developed a number of security design patterns that support reusable security-focused designs tailored for the automotive domain [7] We are also analyzing economic motivations for the perpetrators and socio-technical solutions in the context of automotive cybersecurity [22].

\section{ACKNOWLEDGMENTS}

This work has been supported in part by grants from the NSF ( CNS1305358, and DBI-0939454), Ford Motor Company, General Motors Research, ZF, and the Air Force Research Laboratory (AFRL) under agreement numbers FA8750-16-2-0284 and FA-8750-19-2-0002, and Michigan State University. The U.S. Government is authorized to reproduce and distribute reprints for Governmental purposes notwithstanding any copyright notation thereon. The views and conclusions contained herein are those of the authors and do not necessarily represent the opinions of the sponsors.

\section{REFERENCES}

[1] 2019. http://www.qnx.com

[2] 2019. https://www.exploit-db.com/exploits/21520

[3] GENIVI Alliance. 2013. The GENIVI Alliance. Vol. 28. Online.

[4] Manos Antonakakis, Tim April, Michael Bailey, Matt Bernhard, Elie Bursztein, Jaime Cochran, Zakir Durumeric, J Alex Halderman, Luca Invernizzi, Michalis Kallitsis, et al. 2017. Understanding the mirai botnet. In 26th \{USENIX\} Security Symposium (\{USENIX\} Security 17). 1093-1110.

[5] Pranshu Bajpai, Aditya K Sood, and Richard Enbody. 2018. A key-managementbased taxonomy for ransomware. In 2018 APWG Symposium on Electronic Crime Research (eCrime). IEEE, 1-12.

[6] Alan Calder and Steve G Watkins. 2010. Information security risk management for ISO27001/ISO27002. It Governance Ltd.

[7] B. H. C. Cheng, B. Doherty, N. Polanco, and M. Pasco. 2019. Security Patterns for Automotive Systems. In 2019 ACM/IEEE 22nd International Conference on Model Driven Engineering Languages and Systems Companion (MODELS-C). 5463. https://doi.org/10.1109/MODELS-C.2019.00014

[8] Stephen Cobb. 2017. RoT: Ransomware of Things.

[9] Tiago Cruz, Jorge Barrigas, Jorge Proença, Antonio Graziano, Stefano Panzieri, Leonid Lev, and Paulo Simões. 2015. Improving network security monitoring for industrial control systems. In 2015 IFIP/IEEE International Symposium on Integrated Network Management (IM). IEEE, 878-881.

[10] Mahmoud Hashem Eiza and Qiang Ni. 2017. Driving with sharks: Rethinking connected vehicles with vehicle cybersecurity. IEEE Vehicular Technology Magazine 12, 2 (2017), 45-51.

[11] Pablo L Gallegos-Segovia, Jack F Bravo-Torres, Víctor M Larios-Rosillo, Paúl E Vintimilla-Tapia, Iván F Yuquilima-Albarado, and Juan D Jara-Saltos. 2017. Social engineering as an attack vector for ransomware. In 2017 CHILEAN Conference on Electrical, Electronics Engineering, Information and Communication Technologies (CHILECON). IEEE, 1-6.

[12] Dhruv Garg, Abha Thakral, Tarun Nalwa, and Tanupriya Choudhury. 2018. A Past Examination and Future Expectation: Ransomware. In 2018 International Conference on Advances in Computing and Communication Engineering (ICACCE). IEEE, 243-247.

[13] Alexandre Gazet. 2010. Comparative analysis of various ransomware virii. fournal in computer virology 6, 1 (2010), 77-90.

[14] Mario Gerla, Eun-Kyu Lee, Giovanni Pau, and Uichin Lee. 2014. Internet of vehicles: From intelligent grid to autonomous cars and vehicular clouds. In 2014 IEEE world forum on internet of things (WF-IoT). IEEE, 241-246.

[15] Mukund Ghangurde. 2010. Ford SYNC and Microsoft Windows Embedded Automotive Make Digital Lifestyle a Reality on the Road. SAE International fournal of
Passenger Cars-Electronic and Electrical Systems 3, 2010-01-2319 (2010), 99-105.

[16] Andy Greenberg. 2015. Hackers remotely kill a jeep on the highway-With me in it. Wired 7 (2015), 21.

[17] Julio Hernandez-Castro, Edward Cartwright, and Anna Stepanova. 2017. Economic analysis of ransomware. Available at SSRN 2937641 (2017).

[18] Ben Herzog and Yaniv Balmas. 2016. Great crypto failures. (2016).

[19] Dan Hildebrand. 1992. An Architectural Overview of QNX.. In USENIX Workshop on Microkernels and Other Kernel Architectures. 113-126.

[20] Dirk Inghels, Wout Dullaert, Birger Raa, and Grit Walther. 2016. Influence of composition, amount and life span of passenger cars on end-of-life vehicles waste in Belgium: A system dynamics approach. Transportation Research Part A: Policy and Practice 91 (2016), 80-104.

[21] Daniel Jacobs, Kim-Kwang Raymond Choo, M-Tahar Kechadi, and Nhien-An Le-Khac. 2017. Volkswagen Car Entertainment System Forensics. In 2017 IEEE Trustcom/BigDataSE/ICESS. IEEE, 699-705.

[22] Jay Kennedy, Thomas Holt, and Betty Cheng. 2019. Automotive cybersecurity: assessing a new platform for cybercrime and malicious hacking. Journal of Crime and fustice 42, 5 (2019), 632-645. https://doi.org/10.1080/0735648X.2019.1692425 arXiv:https://doi.org/10.1080/0735648X.2019.1692425

[23] Amin Kharraz, William Robertson, Davide Balzarotti, Leyla Bilge, and Engin Kirda. 2015. Cutting the gordian knot: A look under the hood of ransomware attacks. In International Conference on Detection of Intrusions and Malware, and Vulnerability Assessment. Springer, 3-24.

[24] Constantinos Kolias, Georgios Kambourakis, Angelos Stavrou, and Jeffrey Voas. 2017. DDoS in the IoT: Mirai and other botnets. Computer 50, 7 (2017), 80-84.

[25] Karl Koscher, Alexei Czeskis, Franziska Roesner, Shwetak Patel, Tadayoshi Kohno, Stephen Checkoway, Damon McCoy, Brian Kantor, Danny Anderson, Hovav Shacham, et al. 2010. Experimental security analysis of a modern automobile. In 2010 IEEE Symposium on Security and Privacy. IEEE, 447-462.

[26] Rob Krten. 1999. Getting started with QNX Neutrino 2: a guide for realtime programmers. PARSE Software Devices.

[27] Hiroki Kuzuno, Shun Inagaki, and Kenichi Magata. 2018. Constructing a Complete Timeline of a Security Incident by Aggregating Reports. In 2018 13th Asia foint Conference on Information Security (AsiafCIS). IEEE, 109-115.

[28] Carl E Landwehr. 1983. The best available technologies for computer security. Computer 7 (1983), 86-100.

[29] Gianpaolo Macario, Marco Torchiano, and Massimo Violante. 2009. An in-vehicle infotainment software architecture based on google android. In 2009 IEEE International Symposium on Industrial Embedded Systems. IEEE, 257-260.

[30] Ian Mann. 2017. Hacking the human: social engineering techniques and security countermeasures. Routledge.

[31] Charlie Miller and Chris Valasek. 2015. Remote exploitation of an unaltered passenger vehicle. Black Hat USA 2015 (2015), 91.

[32] David A Mundie and David M McIntire. 2013. The mal: A malware analysis lexicon. Technical Report. CARNEGIE-MELLON UNIV PITTSBURGH PA SOFTWARE ENGINEERING INST

[33] Aurélien Palisse, Hélène Le Bouder, Jean-Louis Lanet, Colas Le Guernic, and Axel Legay. 2016. Ransomware and the legacy crypto API. In International Conference on Risks and Security of Internet and Systems. Springer, 11-28.

[34] Guy Podjarny and Ory Segal. 2014. Method and apparatus for security assessment of a computing platform. US Patent 8,650,651.

[35] Ronny Richardson and Max M North. 2017. Ransomware: Evolution, mitigation and prevention. International Management Review 13, 1 (2017), 10.

[36] Ivan Ristic. 2013. OpenSSL Cookbook: A Guide to the Most Frequently Used OpenSSL Features and Commands. Feisty Duck.

[37] Chris Roberts. 2007. Biometric attack vectors and defences. Computers \& Security 26, 1 (2007), 14-25.

[38] Christoph Schmittner, Gerhard Griessnig, and Zhendong Ma. 2018. Status of the Development of ISO/SAE 21434. In European Conference on Software Process Improvement. Springer, 504-513.

[39] Aditya K Sood, Pranshu Bajpai, and Richard Enbody. 2018. Evidential Study of Ransomware: Cryptoviral Infections and Countermeasures. ISACA fournal 5 (2018).

[40] CAN Specification. 1991. Version 2.0. Robert Bosch GmbH (1991).

[41] Marko Wolf, R Lambert, T Enderle, and AD Schmidt. 2017. Wanna Drive? Feasible Attack Paths and Effective Protection Against Ransomware in Modern Vehicles. In Proc. Embedded Security in Cars Conference (escar) Europe.

[42] Ibrar Yaqoob, Ejaz Ahmed, Muhammad Habib ur Rehman, Abdelmuttlib Ibrahim Abdalla Ahmed, Mohammed Ali Al-garadi, Muhammad Imran, and Mohsen Guizani. 2017. The rise of ransomware and emerging security challenges in the Internet of Things. Computer Networks 129 (2017), 444-458.

[43] Tao Zhang, Helder Antunes, and Siddhartha Aggarwal. 2014. Defending connected vehicles against malware: Challenges and a solution framework. IEEE Internet of Things journal 1, 1 (2014), 10-21. 